\title{
Port placement planning in robot-assisted coronary artery bypass
}

\section{Citation}

Cannon, J.W., J.A. Stoll, S.D. Selha, P.E. Dupont, R.D. Howe, and D.F. Torchiana. 2003. “Port Placement Planning in Robot-Assisted Coronary Artery Bypass." IEEE Transactions on Robotics and Automation 19 (5) (October): 912-917. doi:10.1109/tra.2003.817502.

\section{Published Version}

doi:10.1109/TRA.2003.817502

\section{Permanent link}

http://nrs.harvard.edu/urn-3:HUL.InstRepos:33884775

\section{Terms of Use}

This article was downloaded from Harvard University's DASH repository, and is made available under the terms and conditions applicable to Other Posted Material, as set forth at http:// nrs.harvard.edu/urn-3:HUL.InstRepos:dash.current.terms-of-use\#LAA

\section{Share Your Story}

The Harvard community has made this article openly available.

Please share how this access benefits you. Submit a story.

Accessibility 


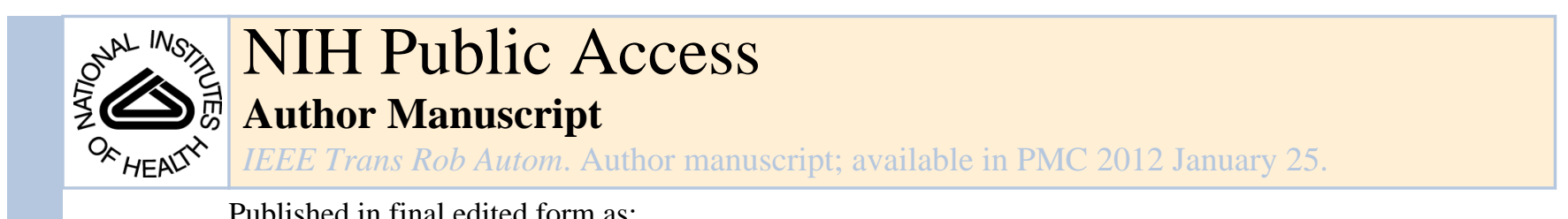

Published in final edited form as:

IEEE Trans Rob Autom. 2003 October ; 19(5): 912-917. doi:10.1109/TRA.2003.817502.

\title{
Port Placement Planning in Robot-Assisted Coronary Artery Bypass
}

\author{
Jeremy W. Cannon, \\ Department of Mechanical Engineering, Massachusetts Institute of Technology, Cambridge, MA \\ 02139 USA (jcannon@massmed.org)
}

Jeffrey A. Stoll, Department of Aerospace and Mechanical Engineering, Boston University, Boston, MA 02215 USA (pierre@bu.edu)

Shaun D. Selha, Department of Aerospace and Mechanical Engineering, Boston University, Boston, MA 02215 USA (pierre@bu.edu)

Pierre E. Dupont, Department of Aerospace and Mechanical Engineering, Boston University, Boston, MA 02215 USA (pierre@bu.edu)

Robert D. Howe, and Division of Engineering and Applied Sciences, Harvard University, Cambridge, MA 02138 USA (howe@deas.harvard.edu)

\section{David F. Torchiana}

Division of Cardiac Surgery, Massachusetts General Hospital, Boston, MA 02114 USA (dtorchiana@partners.org)

\section{Abstract}

Properly selected port sites for robot-assisted coronary artery bypass graft (CABG) improve the efficiency and quality of these procedures. In clinical practice, surgeons select port locations using external anatomic landmarks to estimate a patient's internal anatomy. This paper proposes an automated approach to port selection based on a preoperative image of the patient, thus avoiding the need to estimate internal anatomy. Using this image as input, port sites are chosen from a grid of surgeon-approved options by defining a performance measure for each possible port triad. This measure seeks to minimize the weighted squared deviation of the instrument and endoscope angles from their optimal orientations at each internal surgical site. This performance measure proves insensitive to perturbations in both its weighting factors and moderate intraoperative displacements of the patient's internal anatomy. A validation study of this port site selection was performed. cardiac algorithm also Six surgeons dissected model vessels using the port triad selected by this algorithm with performance compared to dissection using a surgeon-selected port triad and a port triad template described by Tabaie et al., 1999. With the algorithm-selected ports, dissection speed increased by up to $43 \%(p=0.046)$ with less overall vessel trauma. Thus, this algorithmic approach to port site selection has important clinical implications for robot-assisted CABG which warrant further investigation.

\section{(C) 2003 IEEE}

This paper was presented in part at the Telemanipulator and Telepresence Technologies VIII Conference, Newton, MA, October 28, 2001, and in part at the Medical Image Processing and Computer-Assisted Intervention Conference, Utrecht, The Netherlands, October 14-17, 2001 


\section{Keywords}

Medical robotics; port placement; teleoperation

\section{Introduction}

Robotic assistance enables the use of minimally invasive surgical techniques for coronary artery bypass graft $(\mathrm{CABG})$ procedures using instruments inserted through small ports placed between ribs (intercostal spaces) [2]-[4]. During these procedures, the workspace includes both the underside of the chest wall for takedown of the left internal mammary artery (LIMA), as well as the surface of the heart in the middle of the chest, where the LIMA is sutured to a blocked heart vessel (anastomosis) as shown in Fig. 1.

One unique challenge for these port-access $\mathrm{CABG}$ procedures lies in reaching this relatively large workspace through a single triad of intercostal ports (endoscope two instruments). Indeed, initial trials of robot-assisted port-access CABG by the last author confirmed others' findings that port location directly influences access to the surgical sites, dexterity of the surgical instruments, and instrument collisions [3], [5].

\section{A. Prior Work}

Several groups have implemented surgical planning platforms to evaluate various port configurations for these types of procedures. The virtual cardiac surgical planning (VCSP) platform in London, ON, Canada, allows surgeons to manually explore port site selections for individual patients based on a combination of preoperative computer tomography (CT) and magnetic resonance imaging (MRI) images [6], [7]. Another group led by CosteManière has implemented a mathematical algorithm for selecting port locations [8], [9]. This planning algorithm is based on distance to the surgical site, the architecture of the teleoperation system, and several important anatomic interference constraints. These constraints include the ribs, which can be injured by steeply angled instruments, as well as the diaphragm and shoulder.

Guidelines for selecting port sites for these procedures based on clinical experience have also been described [1], [3], [5], [10]. These recommendations are based on external landmarks and assume that these landmarks accurately indicate the patient's internal anatomy which must be manipulated during the surgical procedure.

\section{B. External versus Internal Landmarks}

Similarly, in clinical practice, surgeons estimate the location of the internal surgical sites based on the size of the patient's torso and external landmarks, such as the nipple and sternum. Unfortunately, when the external landmarks do not accurately correspond to the individual patient's internal anatomy due to individual variability or due to imprecise mental estimation, misplaced port sites can result. In these cases, the difficulty of the procedure increases greatly, which can lead to costly delays and possibly even additional incision(s) for the patient if port(s) require resiting.

To address these problems of current approaches, this paper presents a new method for identifying the appropriate port sites for CABG procedures. Rather than using surface landmarks, this method solves the inverse problem: given a set of internal surgical sites and knowledge of the optimal relative instrument and endoscope angles, determine where each port should be positioned in the chest wall. This approach starts by quantifying the desired 
surgical result as a performance measure and then searches over all available solutions to identify the one which is optimal.

The paper is arranged as follows. Section II details the algorithmic approach, followed by an experimental evaluation of the algorithm in Section III. Sensitivity to perturbations in the weighting factors and the internal anatomy is investigated, followed by a surgeon evaluation and comparison with existing port placement techniques. Finally, a discussion of these results leads to several conclusions (Section IV).

\section{Approach}

Difficulties encountered when using suboptimal port sites can be categorized as: 1) inability to reach a surgical site; 2) inability to complete surgical tasks due to the relative orientations of the instruments, endoscope, and surgical site; 3 ) internal instrument/scope collisions; 4) robot singularities; 5) robot joint limits; and 6) robot arm collisions.

As shown in Fig. 2, the port sites act as fulcrums for the instruments and endoscope. Consequently, the port sites, together with motions at the internal surgical sites, define the motion of the shafts exterior to the body. Thus, the overall kinematics problem can be decomposed into two independent parts: port placement to optimize performance at the surgical sites (difficulties 1-3), and robot placement to ensure unimpeded motion exterior to the body (difficulties 4-6). This paper examines port placement only as the issues associated with robot placement for this particular robotic system have been resolved through the laboratory experience of the authors and others [1].

Laboratory studies by the last author have shown the importance of the following four performance criteria for assessing the quality of a given port location.

1. Preserve the surgeon's intuition by maintaining the relative orientation of the surgeon's hands and eyes as in open surgical procedures.

2. Employ relative angles between the instruments, endoscope, and surgical site that facilitate the specific intraoperative surgical task (e.g., dissecting or suturing).

3. Avoid internal interference (collisions) between the instruments, endoscope, and tissue.

4. Allow clearing of the endoscope lens with a gravity-fed drip system.

\section{A. Surgical Site Coordinate Frame}

Given these performance criteria, the optimal orientations of the instruments and endoscope can be defined with respect to a coordinate frame with the origin placed at each internal surgical site (Fig. 3). This of coordinate frame is similar to that described in [1], which satisfies criteria 1 above by mimicking the relative angles of approach of the surgeon's hands and viewpoint during open surgery.

For the $\mathrm{CABG}$ considered here, the $y$ axis is directed vertically up-ward to approximate the surface normal at the coronary artery, and the axis is chosen parallel to the patient's spine, directed toward the head. For the surgical sites along the LIMA, the normal is approximated vertically downward.

The two instruments lie on a plane (the instrument plane) with angle of elevation $\gamma$ referenced from the $z$ axis. Within the instrument plane, the instruments are oriented at yaw angles of $\theta_{1}$ and $\theta_{\mathrm{r}}$ with respect to the negative and positive $x$ axis, respectively. For 
endoscope orientation, $\varphi_{\mathrm{e}}$ and $\varphi_{\mathrm{a}}$ are the elevation and azimuthal angles, respectively, while $\varphi_{0}$ is the fixed angle of the endoscope lens $\left(\varphi_{0}=30^{\circ}\right.$ here $)$.

To date, a complete analytical derivation of optimal orientation angles based on such criteria as dexterity, workspace, and interference has not been undertaken. However, clinical studies of orientation angles have been performed by the authors and others [1], [11]-[13]. As described in Sections II-B-D, the resulting preferred orientation angles can be clearly tied to criteria 2-4 above. The optimal values appear in Table I. Clinical experience has shown that acceptable performance is obtained in a working range of $\pm 15^{\circ}$ around these values.

\section{B. Optimal Instrument Angles}

Instrument orientation is described by the angle of elevation of the instrument plane and by the yaw angles within that plane. Instrument yaw angle has been addressed in the literature by several authors with recommended instrument separation angles from $45^{\circ}$ to $90^{\circ}$ for surgical tasks ranging from simple cauterization to suturing [1], [11]-[13]. Our experience suggests that for CABG procedures which involve both dissection and suturing, $\theta_{\mathrm{opt}}=60^{\circ}$ This value can be justified using criteria 2 (task facilitation) and 3 (collision avoidance) explained as follows.

Using the fixed offset grasper of Fig. $4, \theta_{\mathrm{opt}}=60^{\circ}$ provides the greatest flexibility for interaction with both the tissue at the surgical site and the other instrument. As shown, when the grasper tip is normal to the tissue in the tool plane, it can grasp tissue precisely. If the instrument is rotated by $180^{\circ}$ about its axis (not shown), the offset tip is now normal to the second instrument within the tool plane permitting precise interaction between the instruments. Furthermore, collision avoidance is achieved by maximizing the angles of separation between each instrument and the tissue, which is achieved by using $\theta_{\mathrm{opt}}=60^{\circ}$.

Ideal instrument plane elevation angle has been reported as $30^{\circ}$ in [1]. Our experience indicates that for suturing, the elevation angle should be opt $=45^{\circ}$. This angle, combined with gripper offset angle and curvature of the suturing needle, provides the surgeon with a broad range of approach angles with respect to the surface normal of the surgical site. Larger angles of elevation rotate the instrument plane such that it encroaches on the endoscope, making interference more likely.

For CABG, suturing the anastomosis is far more difficult and critical than LIMA takedown. From Fig. 1, it is clear that the desired value of instrument plane elevation angle $\gamma_{\mathrm{opt}}=45^{\circ}$, cannot be achieved simultaneously for both LIMA takedown and anastomosis from a single triad of ports. Consequently, the instrument elevation angle for takedown can be reduced to accommodate a more optimal approach for suturing. During LIMA takedown, experience indicates that an elevation angle $\gamma_{\mathrm{opt}}=-20^{\circ}$ allows the surgeon to separate the vessel from the chest wall and surrounding tissues effectively (the normal for the LIMA takedown points along the negative y axis of Fig. 3).

\section{Optimal Endoscope Angles}

Endoscope orientation is described by its azimuthal and elevation angles. An azimuthal angle near $\varphi_{\mathrm{a}}=0^{\circ}$ preserves intuition by maintaining the viewpoint between the instruments for both LIMA takedown and anastomosis. It also maximizes the distance between the endoscope and the instruments along their length and minimizes the length of endoscope inserted into the chest cavity. The latter two reduce interference between the endoscope and instruments. For the large variation in $\varphi_{\mathrm{a}}$ during LIMA takedown (see Fig. 1), the value $\varphi_{\mathrm{a} \text {,opt }}=0^{\circ}$ also serves to provide a uniform field of view along the vessel length while limiting parallax effects. 
The elevation angle of the endoscope $\varphi_{\mathrm{e}}$, together with the constant offset $\varphi_{\mathrm{o}}=30^{\circ}$, determine the angle between the viewing axis and the surface normal of the surgical site. Our laboratory trials have shown that a viewpoint normal to the surface closely simulates open surgery and is the most effective choice for suturing. This suggests an optimal elevation angle of $\varphi_{\mathrm{e} ; \mathrm{opt}}=60^{\circ}$. To satisfy criteria 4 above, in this specific study, $\varphi_{\mathrm{e} ; \mathrm{opt}}=52^{\circ}$ is used for anastomosis, giving a viewing angle of $82^{\circ}$ (Fig. 5).

As with optimal instrument orientations, the anatomy of the CABG procedure (Fig. 1) precludes achieving the same endoscope elevation angle for both anastomosis and LIMA takedown. Since LIMA dissection is the easier task, laboratory trials were undertaken to determine the minimum elevation angle allowing effective LIMA dissection. With the endoscope's lens pointed upward, an elevation angle, $\varphi_{\mathrm{e} ; \mathrm{opt}}=7^{\circ}$, combines with the $30^{\circ}$ offset to give a $23^{\circ}$ viewing angle (Fig. 6). Viewing angles greater than this require further insertion of the endoscope into the chest cavity, which increases the probability of internal collisions.

\section{Performance Measure}

A surgical site, together with a triad of ports lying in the intercostal spaces, defines the instrument and endoscope angles of Fig. 3. To judge the optimality of this port triad, these angles can be assembled into a vector $\psi$ and compared with the optimal values given as $\psi_{\mathrm{opt}}$ in Table I. Many surgical procedures, including CABG, involve several surgical sites which must be accessed using the same port triad. Thus, a performance measure $J$ can be defined as the sum of the weighted squared "distance" of the instruments and endoscope from their optimal orientation angles over surgical sites

$$
J=\sum_{i=l}^{n} K_{i}\left(\psi_{i}-\psi_{\mathrm{opt}}\right)^{T} W\left(\psi_{i}-\psi_{\mathrm{opt}}\right) .
$$

A triad of ports is considered optimal to another set if it has a smaller performance measure. Here, $W$ is a diagonal weighting matrix by which the relative importance of the angles can be taken into account, and $k_{\mathrm{i}}$ is the weighting factor for the $i$ th surgical site. Clinically determined values of $W$ appear in Table I.

For CABG planning, five surgical sites were selected, $(n=5)$ with one at the coronary artery and four more along the LIMA. Equal weighting for the coronary site and the LIMA as a whole is achieved by assigning $k=1$ for the coronary and $k=0.25$ for each LIMA site.

Given a list of surgeon-selected feasible port sites in the intercostal spaces, together with a list of surgical sites (defined by their $\{x, y, z\}$ coordinates and surface normal), the triad of ports that minimizes $J$ is accepted as optimal. The ribs, diaphragm, and other anatomic structures limit candidate port sites to a modest number $(<200)$. Because the weighting matrix $W$ is diagonal, the ranking of each port in the triad is uncoupled; thus, an exhaustive comparison of $m$ feasible ports requires only $3 m$ evaluations of (1).

\section{Experimental Evaluation}

The port placement algorithm was assessed for sensitivity to the choice of angle weighting factors and to perturbations in surgical site location. A validation study of these algorithmic port selections was then undertaken. 


\section{A. Sensitivity Analysis}

The proposed approach assumes that a set of angle weighting factors $w_{i i}$, can be identified such that (1) accurately measures the optimality of both the instruments and the endoscope. This performance measure furthermore ranks port triads based on patient position within the imaging device, which may prove inaccurate at the time of surgery due to relative shifts of the internal anatomy.

In analyzing weighting factor sensitivity, only the three ratios of weights corresponding to the two instruments $\left(\theta_{r} / \gamma_{r}=\theta_{l} / \gamma_{l}=1.4\right.$ in Table I $)$ and the endoscope $\left(\varphi_{\mathrm{e}} \varphi_{\mathrm{a}}=1.67\right.$ in Table I) need be considered because the weighting matrix is diagonal. These three ratios were varied simultaneously over the range $0.5-2$, and the optimal port triads were computed for a dense intercostal set of 134 feasible ports. For the left instrument, all choices of weighting factors yield the same port site. For the endoscope and right instrument, the variation in weighting factor produces pairs of adjacent ports lying in a single intercostal space. Given tissue compliance, these adjacent ports were essentially equivalent. Thus, port selection is robust to the choice of weighting factors.

In assessing the sensitivity to anatomic shifts, relative shifts in the LIMA are less likely as it is embedded in the chest wall. Thus, this analysis focused on the coronary site. A sphere of radius $1 \mathrm{~cm}$ centered at the original coronary site was defined, and optimal port triads were computed for surgical sites lying on the surface of this sphere. The results indicate robustness to relative shifts in surgical site similar to that observed for variations in weighting factors. Specifically, port site location varied by no more than $1 \mathrm{~cm}$.

\section{B. Experimental Comparison}

To compare algorithm-selected ports with existing port site selection approaches, six staff cardiac surgeons were recruited to perform robot-assisted LIMA takedown and coronary dissection using a torso model. Three port triads were used by each surgeon representing the following distinct scenarios: 1) ports selected by someone trained specifically in port-access CABG (LIT); 2) ports selected by staff cardiac surgeons new to the port-access CABG procedure (SURG); and 3) ports selected based on the precise internal anatomy and the optimal relative orientations of the instruments and endoscope with respect to all surgical sites (ALG) (Fig. 7).

For this study, the literature-based port site triad (LIT) was taken from the guidelines given by Tabaie [1]. A second triad of port sites (SURG) was compiled from input by three cardiac surgeons experienced in thoracoscopic and minimally invasive cardiac surgical techniques, but with no formal training in performing robot-assisted CABG. The port sites recommended by these surgeons for the Right Instrument and Endoscope positions were tightly clustered within the third and fifth interspaces, respectively. For the left instrument, one surgeon recommended placement in the sixth interspace, while the others recommended placement in the seventh interspace. An average of the latter sites was used for the SURG triad.

Finally, the algorithm presented in this paper was implemented to obtain the third triad (ALG). A preprocedure CT was taken with the skeleton model placed in the operative position and the model vessels mounted in place. This image was then segmented (3D Slicer, 1.3.0, Brigham \& Women's Hospital, Boston, MA and Massachusetts Institute of Technology (MIT), Cambridge, MA) to show the soft tissue layer (neoprene rubber), the ribs, and the model vessels (Fig. 7). Within a user interface (described in [14]), a total of 52 distinct clinically safe candidate port sites were identified in the intercostal spaces by an experienced surgeon. Five internal surgical sites were also selected, including one coronary site and four LIMA sites. 
The surgical evaluation task required dissection of a model vessel adapted from [15] (Fig. 8). The model consists of a 3-mm diameter "vessel" of stiff clay (Mortite Rope Caulk, EFI, Westborough, MA) rolled into a "soft tissue matrix" of modeling dough which was then shrink wrapped. Two of these vessels were mounted at the extremes of the LIMA (proximal LIMA and distal LIMA), and a third placed at the site of the coronary artery.

These dissection tasks were all completed with a ZEUS telerobotic surgical system (formerly Computer Motion, Inc., Goleta, CA). Using a harmonic scalpel (R instrument) and a fixed-offset grasper (L instrument), each surgeon performed three dissections (proximal LIMA, distal LIMA, and coronary) for each of the port triads (LIT, SURG, and ALG).

Performance measures included dissection speed for each vessel location and a measure of vessel damage indicated by length of gouges in the clay of $>1.5 \mathrm{~mm}$ deep or complete vessel transection. Internal instrument conflicts violating criteria 2 and 3 of Section II were also noted. Statistical analysis was based on a two-tailed Wilcoxon signed rank test (SPSS 10.1.0, Chicago, IL) with $p \leq 0.05$ considered statistically significant.

\section{Results of Experimental Comparison}

Comparisons were made between ALG versus LIT and ALG versus SURG performance (Figs. 9 and 10). Dissection speed for the proximal LIMA was the same for all triads. For the distal LIMA, using the ALG triad, dissection speed increased by $54 \%$ versus LIT ( $p=$ $0.080)$ and $26 \%$ versus SURG $(p=0.25)$. Coronary dissection speed increased by $39 \%$ using the ALG triad versus LIT $(* p=0.028)$ and $43 \%$ versus SURG $(* p=0.046)$. Mean dissection speed increased by $26 \%$ versus LIT $(* p=0.028)$ and $30 \%$ versus SURG $(* p=$ 0.046).

For the proximal LIMA, gouge length decreased by $44 \%$ for ALG versus LIT $(p=0.27)$ and by $54 \%$ versus SURG $(p=0.075)$, although neither reduction was statistically significant. Distal LIMA injuries were reduced by $87 \%$ for ALG versus SURG $(p=0.080)$. Coronary gouge length was not significantly less for either ALG versus LIT or for ALG versus SURG ( $p=0.66$ and 0.27 , respectively). Mean gouge lengths for ALG versus LIT were not statistically different $(p=0.27)$, while mean gouge length was $70 \%$ less for ALG versus SURG $(* p=0.046)$.

Each triad had at least one instrument or line-of-sight conflict during the 18 vessel dissections. The most conflicts occurred with the LIT triad $(n=3)$, with the SURG and ALG triads recording only one conflict each.

\section{Discussion}

This algorithm, based on a quantitative measure of port optimality, appears to offer significant improvements in surgical task efficiency and quality for CABG procedures over surgeon-selected or literature-based port triads. The sensitivity analysis indicates that the proposed performance measure is insensitive to angle weighting factors. Furthermore, the identified port sites remain valid even for moderate shifts in patient anatomy.

The model study for LIMA and coronary dissection was designed to resemble the actual clinical procedure of robot-assisted $\mathrm{CABG}$ while standardizing the approach to permit meaningful performance comparisons. By using staff cardiac surgeons with extensive training in thoracoscopic techniques, including use of a surgical teleoperation system as subjects, the results of this validation indicate that algorithmic port site selection can improve procedure performance at even the highest level of clinical skill. 
Furthermore, this method of uniquely defining a performance measure for individual surgical tasks permits the application of this approach to new robot-assisted procedures so long as optimal angles and weighting factors can be identified [16]. Ultimately, this type of algorithm may facilitate optimal surgical performance earlier in the learning curve for these procedures.

This study did not evaluate any extreme cases of potential patient size or situations where anatomic shifts are large or unknown (as with lung collapse and gas insufflation). To determine the effects of lung collapse and insufflation, this port site selection algorithm could be applied to registered sequential images while using varying levels of chest insufflation. Both of these examples (anatomic extremes and chest insufflation) represent important elements of future validation work.

Thus, pending further validation, this algorithmic approach may improve the efficiency and safety of robot-assisted CABG by optimizing placement of the instrument and endoscope ports. Implementation of this algorithm for robot-assisted CABG and similar algorithms for other robot-assisted procedures could assist surgeons in transitioning to telesurgical techniques while ensuring the best possible clinical outcome from these procedures.

\section{Acknowledgments}

The authors gratefully acknowledge the technical assistance of J. Titus and Dr. J. White in the MGH Cardiovascular Surgery Laboratory.

The work of J. W. Cannon was supported by the National Institutes of Health (NIH) under Grant F32 HL68404-01. The work of J. A. Stoll was supported by The Center for Integration of Medicine and Innovative Technology. The work of S. D. Selha was supported by a GEM Fellowship.

\section{References}

[1]. Tabaie HA, Reinbolt JA, Graper WP, Kelly TF, Connor MA. Endoscopic coronary artery bypass graft (ECABG) procedure with robotic assistance. Heart Surgery Forum. 1999; 2:310-315. [PubMed: 11276493]

[2]. Boyd WD, et al. Closed-chest coronary artery bypass grafting on the beating heart with the use of a computer-enhanced surgical robotic system. J. Thoracic Cardiovascular Surgery. 2000; 120:807-809.

[3]. Kappert U, et al. Development of robotic enhanced endoscopic surgery for the treatment of coronary artery disease. Circulation. 2001; 104(Suppl. 1):102-107. [PubMed: 11435346]

[4]. Argenziano, M. Columbia-Presbyterian minimally invasive and robotic cardiac surgery program; Engineering the Future of Surgery: Symp. for Surgeons and Engineers; New York, NY. 2002;

[5]. Loulmet D, et al. Endoscopic coronary artery bypass grafting with the aid of robotic assisted instruments. J. Thoracic Cardiovascular Surgery. 1999; 118:4-10.

[6]. Lehmann, G., et al. Toward dynamic planning and guidance of minimally invasive robotic cardiac bypass surgical procedures; Proc. MICCAI 2001; Utrecht, The Netherlands. 2001; p. 368-375.

[7]. Chiu A, Day D, Drangova M, Boyd D, Peters T. 3-D image guidance for minimally invasive robotic coronary artery bypass. Heart Surgery Forum. 2000; 3:224-231. [PubMed: 11074977]

[8]. Adhami, L.; Coste-Manière, E.; Boissonnat, J-D. Planning and simulation of robotically assisted minimal invasive surgery; Proc. Medical Image Computing and Computer Assisted InterventionMICCAI 2000; Pittsburgh, PA. 2000; p. 624-633.

[9]. Coste-Manière, E., et al. Optimized port placement for the totally endoscopic coronary artery bypass grafting using the Da Vinci robotic system. In: Russ, D.; Singh, S., editors. Lecture Notes in Control and Information Sciences, Experimental Robotics VII. Vol. 271. Springer-Verlag; Berlin: 2001. p. 199-208.

[10]. Damiano RJ, et al. Initial prospective multicenter clinical trial of robotically-assisted coronary artery bypass grafting. Ann. Thoracic Surgery. 2001; 72:1263-1269. 
[11]. Oppenheimer P, et al. Laparoscopic surgical simulator and port placement study. Studies Health Technol. Inform. 2000; 70:233-235.

[12]. Hanna GB, Shimi S, Cuschieri A. Optimal port locations for endoscopic intracorporeal knotting. Surgical Endoscopy. 1997; 11:397-401. [PubMed: 9094288]

[13]. Hanna GB. Influence of direction of view, target-to-endoscope distance and manipulation angle on endoscopic knot tying. British J. Surgery. 1997; 84:1460-1464.

[14]. Selha, S.; Dept. Aero. Mech. Eng., Boston Univ.. Master's thesis. Boston, MA: 2002. Dexterity optimization through port placement in robot-assisted minimally invasive surgery.

[15]. Wagner, C.; Stylopoulos, N.; Howe, R. The role of force feedback in surgery: Analysis of blunt dissection; Proc. 10th Int. Symp. Haptic Interfaces for Virtual Environment and Teleoperator Systems; Orlando, FL. 2002; p. 73-79.

[16]. Cannon J, Howe R, Dupont P, Triedman J, Marx G, del Nido P. Application of robotics in congenital cardiac surgery. Proc. Seminar Thoracic Cardiovascular Surgery: Pediatric Cardiac Surgery Ann. 2003; 6:72-83. 


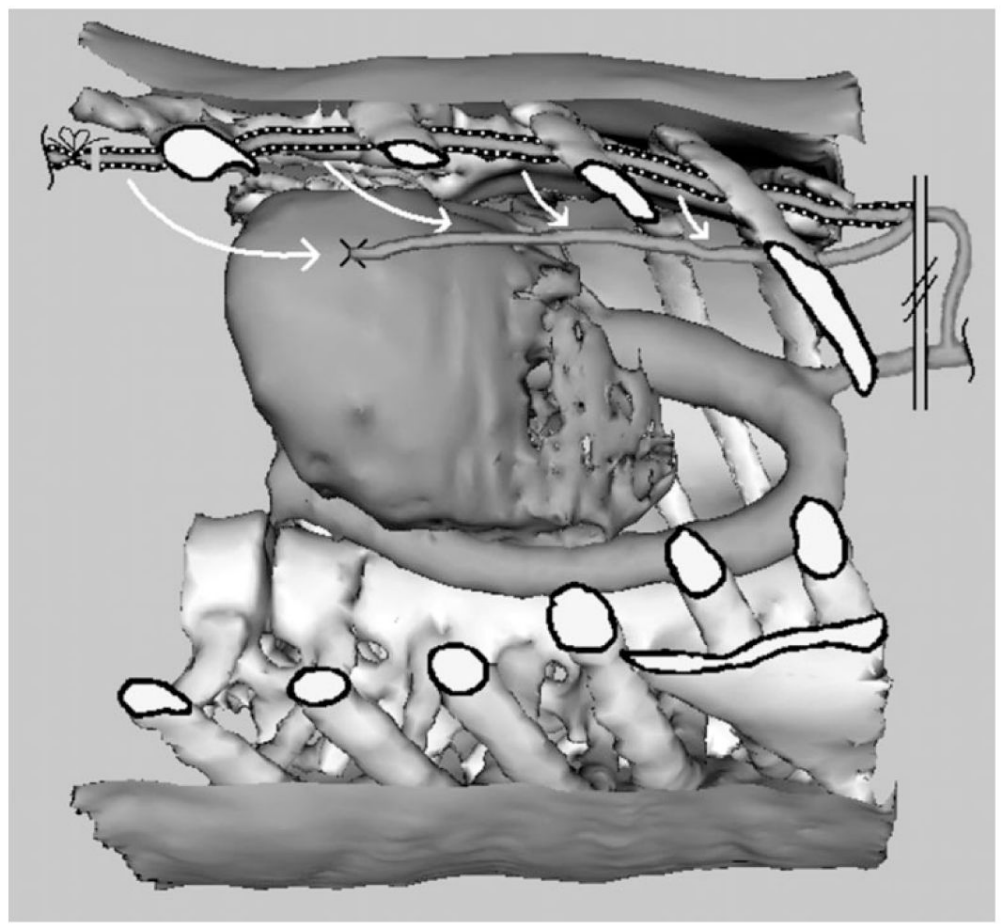

Fig. 1.

Anatomy of the CABG procedure as seen through a cutaway window in the chest wall. The LIMA is shown prior to dissection on the underside of the chest wall (dashed) and after dissection sutured onto a coronary artery (solid). 


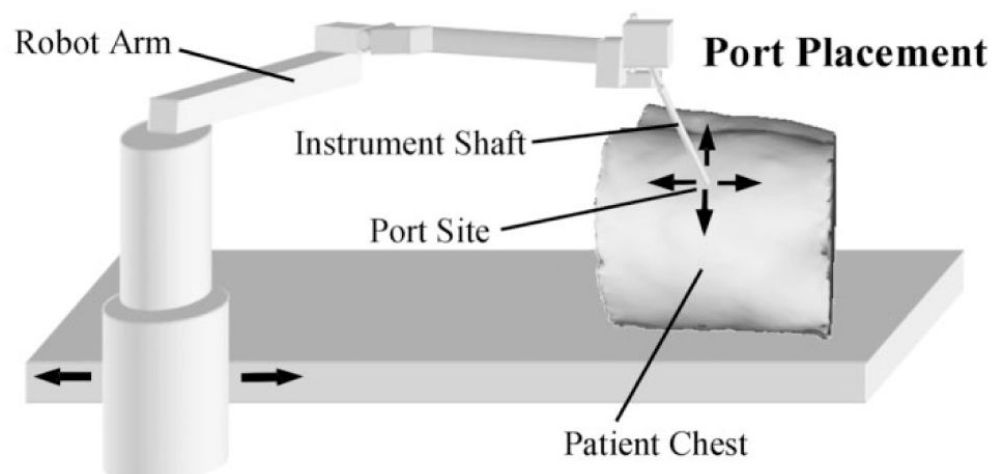

\section{Robot Placement}

Fig. 2.

Port placement versus robot placement. Arrows on patient chest indicate possible locations for placing a port site. Arrows at the robot indicate possible locations of robot base. 


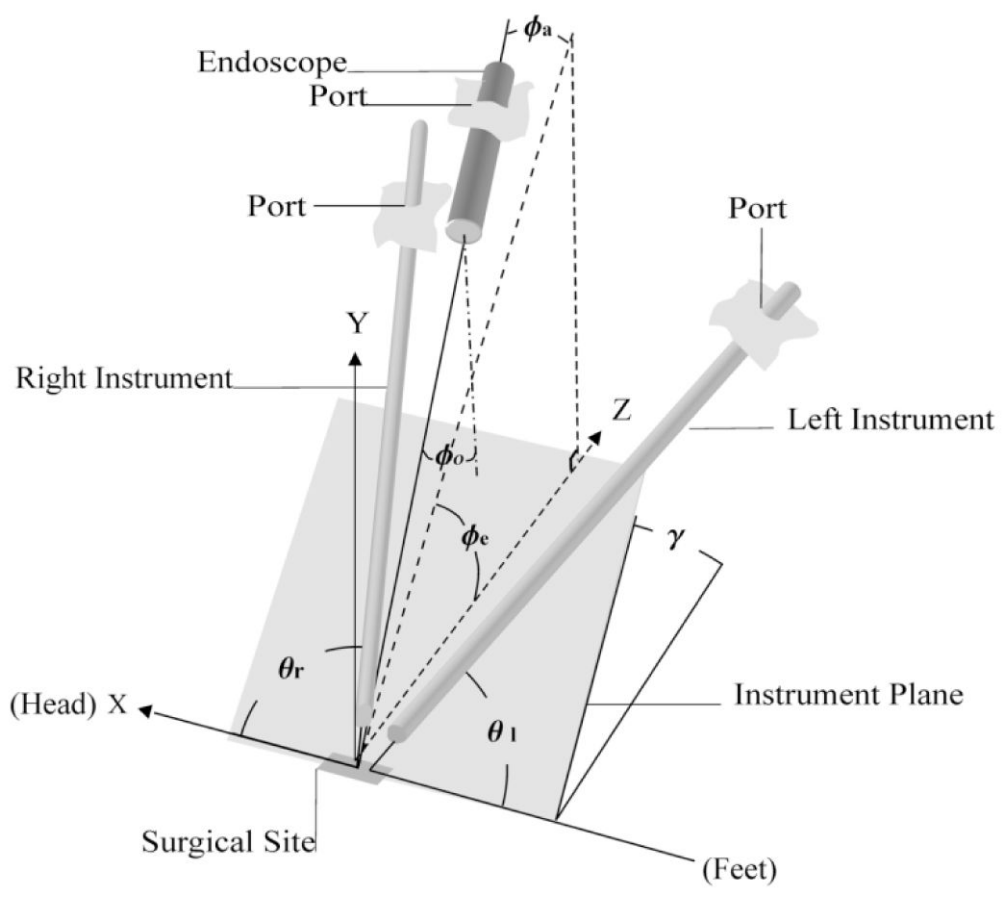

Fig. 3.

Surgical site coordinate frame. 


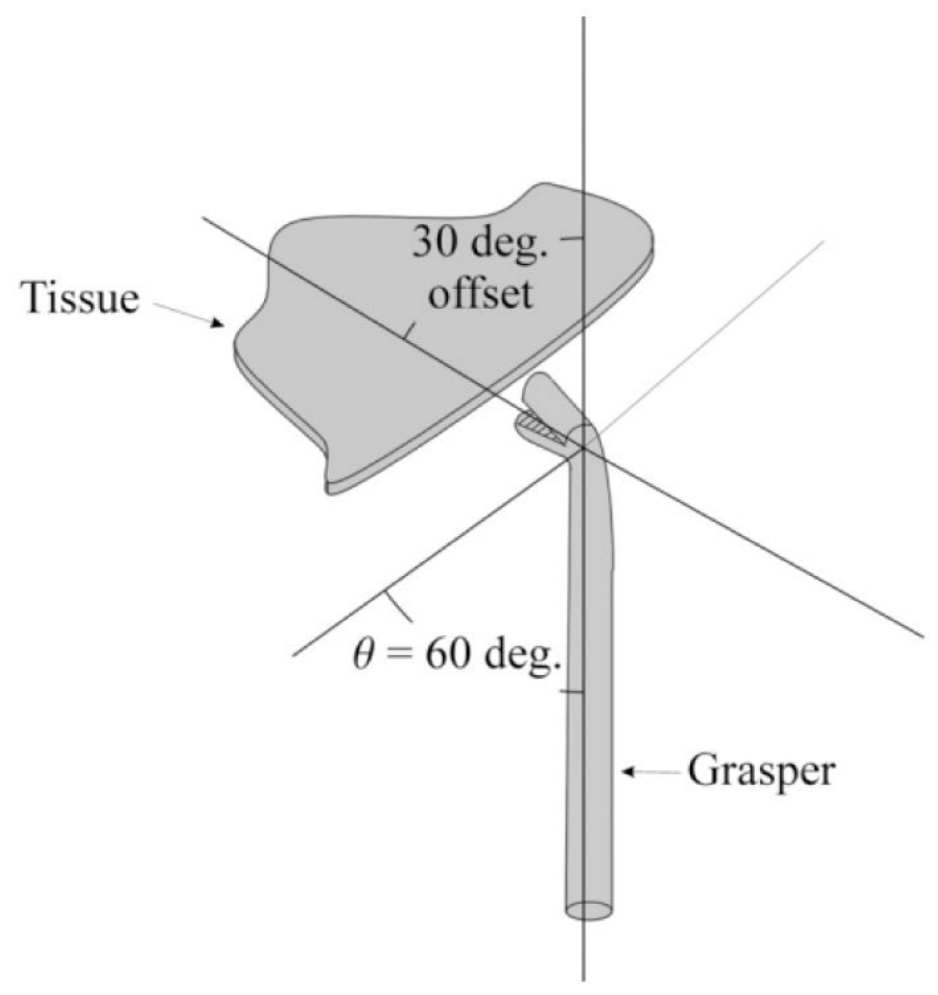

Fig. 4.

Tissue manipulation with a fixed-offset grasper. 


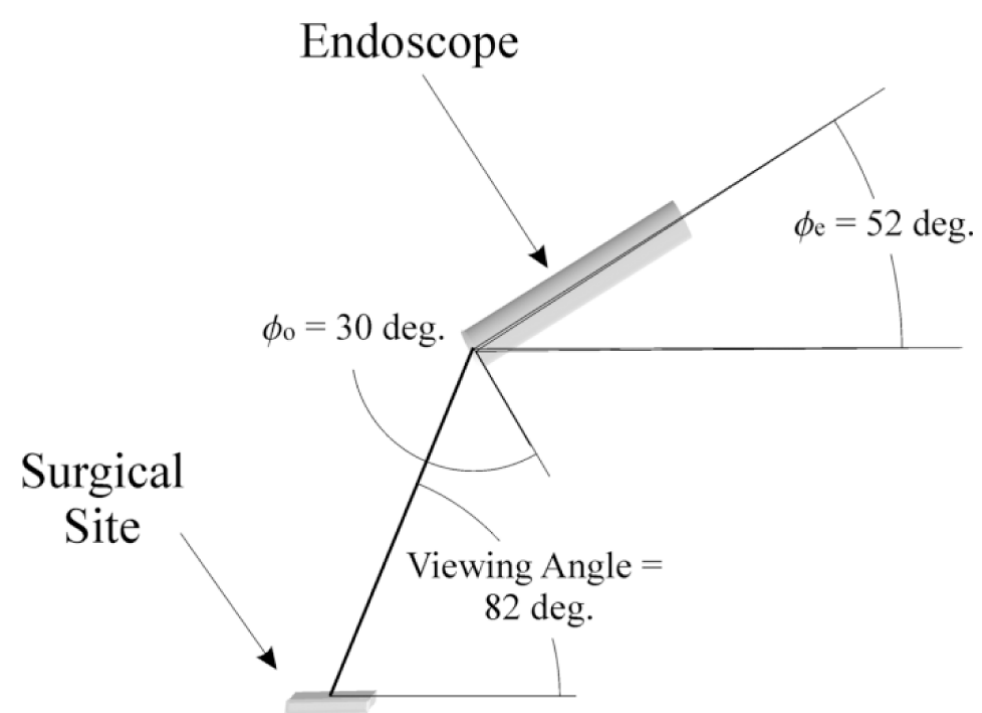

Fig. 5.

Optimal endoscope orientation for anastomosis. 


\section{Surgical Site}

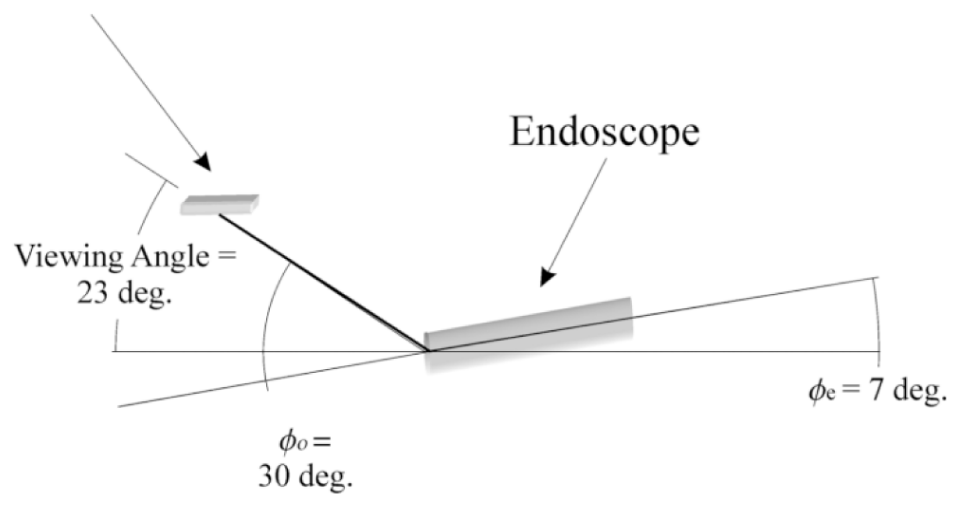

Fig. 6.

Optimal endoscope orientation for LIMA takedown. 


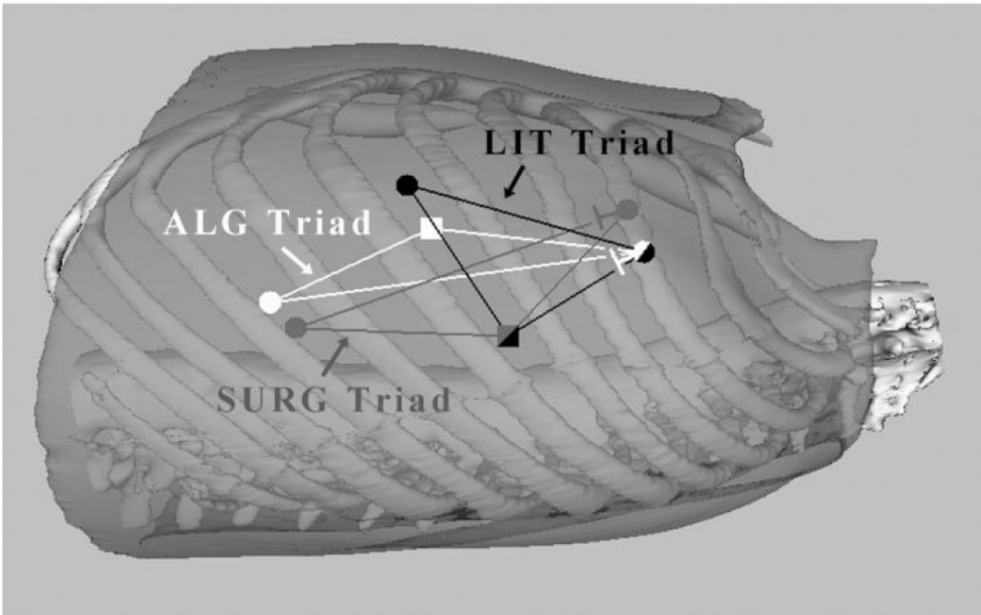

Fig. 7.

Illustration of port triads. Square endoscope port, circle instrument ports. 


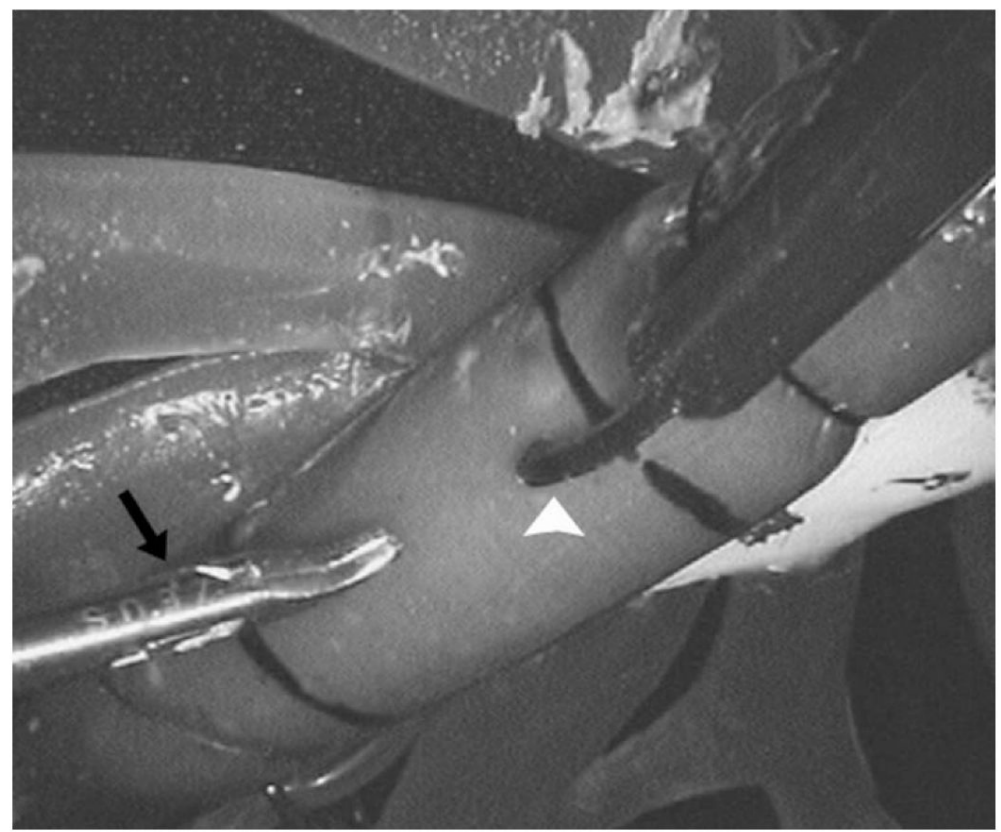

Fig. 8.

LIMA takedown task for port placement evaluation. This picture shows the distal LIMA setup from the endoscope view. A fixed-offset grasper (black arrow) and a harmonic scalpel (white arrowhead) are poised to dissect the model vessel from the surrounding soft tissue. 


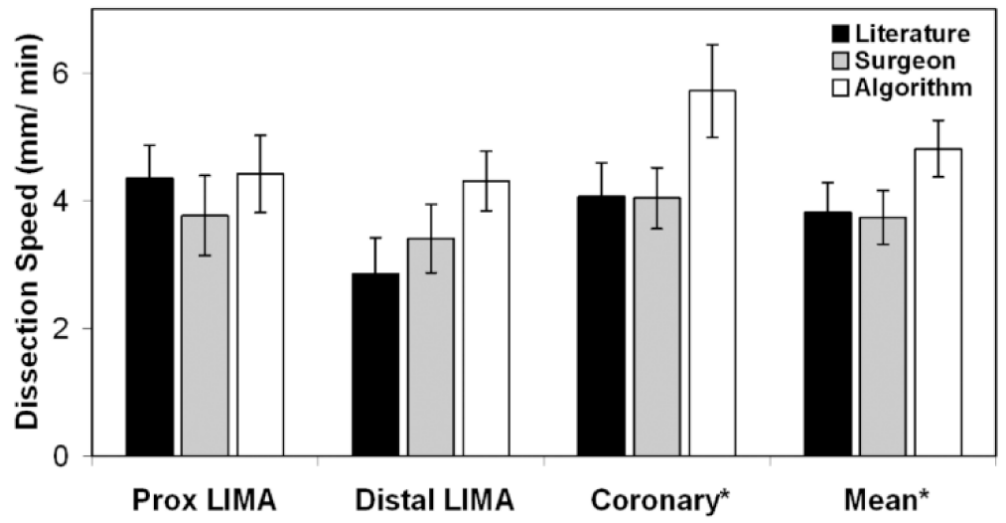

Fig. 9.

Dissection speed. ALG versus LIT and ALG versus SURG, $p<0.05$. 


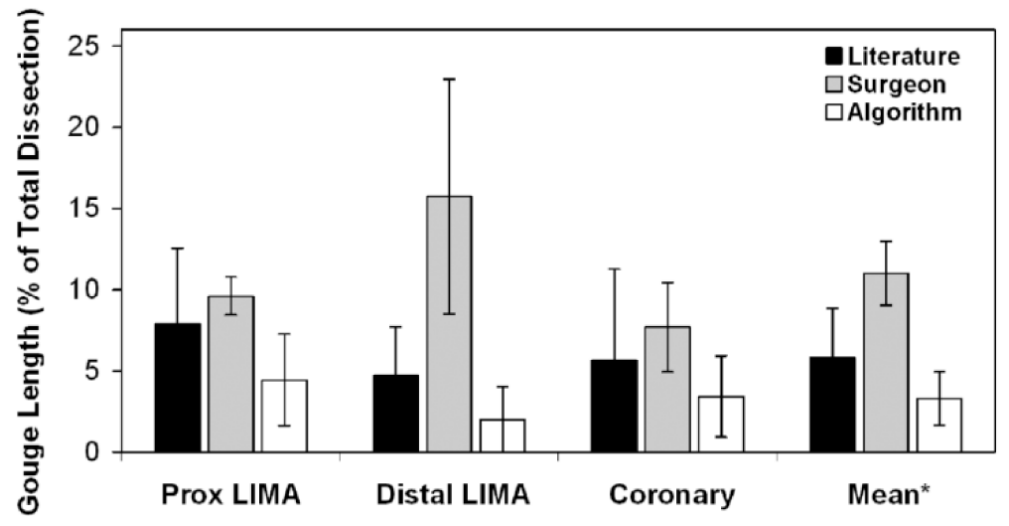

Fig. 10.

Gouge length. * ALG versus SURG, $p<0.05$. 


\section{TABLE I}

Weighting Factors and Optimal Angles

\begin{tabular}{cccc}
\hline Angle & $\begin{array}{c}\text { Angle Weighting } \\
\text { Factors } \boldsymbol{W}_{\boldsymbol{H}}\end{array}$ & \multicolumn{2}{c}{$\boldsymbol{\psi}_{\text {opt }}$ (degrees) } \\
LIMA takedown & Anastomosis \\
\hline$\theta_{r}$ & 1.40 & 60 & 60 \\
$\gamma_{r}$ & 1 & -20 & 45 \\
$\theta_{l}$ & 1.40 & 60 & 60 \\
$\gamma_{l}$ & 1 & -20 & 45 \\
$\varphi_{e}$ & 1.67 & 7 & 52 \\
$\varphi_{a}$ & 1 & 0 & 0 \\
\hline
\end{tabular}

Endoscope offset angle $\varphi_{\mathbf{O}} 30^{\circ}$ 